\title{
Right coronary artery spasm, QRS-ST-T "shark fin" pattern, and sudden cardiac death. A case report.
}

\author{
Anthony Kechichian ${ }^{1}$ and Antoine Sarkis ${ }^{2}$ \\ ${ }^{1}$ Hôtel-Dieu de France \\ ${ }^{2}$ Hotel-Dieu De France
}

September 16, 2020

\begin{abstract}
Malignant ventricular arrhythmic storm and sudden cardiac death (SCD) following coronary vasospasm (CVS) are relatively uncommon. On another note, a specific pattern, the QRS-ST-T "shark fin" pattern on ECG, is known to be a high-risk pattern and is associated with a very poor prognosis in the context of coronary artery occlusion. Data reporting the association of CVS-induced SCD and specific high-risk ECG patterns is scant. Herein we present a case of a patient with right coronary artery (RCA) spasm who presented in sudden cardiac death and a "shark fin" pattern on ECG. Primary stenting of the residual lesion after intra-coronary nitroglycerin was performed. The patient received optimal medical therapy and an intracardiac defibrillator. Lowering the threshold for defibrillator implantation may be reasonable in CVS-induced ventricular arrhythmias particularly in the presence during vasospasm of high-risk ECG features such as the "shark fin" pattern.
\end{abstract}

\section{Introduction}

Prinzmetal's angina also known as variant angina or angina inversa refers to an equivalent of the exertion angina, which frequently occurs at rest. The phenomenon was first described in 1959 by Prinzmetal et al who adopted this terminology to define the transient and significant narrowing of the coronary lumen associated with ST-segment elevation and presumably due to vasospasm ${ }^{1}$. Although various hypotheses ranging from endothelial dysfunction and primary vessel hyperreactivity to key triggering stimuli have been studied, our understanding of the pathophysiology and complications of coronary vasospasm (CVS) remain somewhat limited $^{2}$. Sudden cardiac death (SCD) related to coronary spasm is probably underestimated and could account for $2 \%$ of all $\mathrm{SCD}^{3}$. On another hand, several reports have noted an association between coronary artery occlusion and some high-risk electrocardiogram (ECG) patterns ${ }^{4,5}$, such as the "shark fin" pattern also known as "giant R waves" or "triangular QRS-ST-T waveform" (TW) (Fig. 1). However, to our best knowledge, specific ECG patterns in the context of CVS-induced ventricular lethal arrhythmias have not been previously reported.

We report the case of a patient with multiple cardiovascular risk factors, presenting with SCD related to right coronary artery (RCA) spasm, preceded by a QRS-ST-T "shark fin" pattern on ECG.

\section{Case report}

A 69 year-old male patient known to be hypertensive, diabetic, alcoholic, and a heavy smoker, presented to the emergency department of our hospital in cardiac arrest, following repetitive chest pain episodes at rest. Two weeks earlier, he experienced similar chest pain with syncope, and a coronary angiogram at that time showed 60 to $70 \%$ narrowing in the mid RCA and in the proximal left anterior descending (LAD) artery. He was advised to undergo a fractional flow reserve (FFR) evaluation in order to decide for a stent implantation, but he left the hospital against medical advice. 
Upon his admission in sudden arrest, ECG was in ventricular fibrillation. Nevertheless, repeated ECGs during resuscitation showed a transient ST-segment elevation in the inferior leads, with a QRS-ST-T "shark fin" pattern and frequent $R / T$ ventricular premature contractions (VPCs) (Fig. 2). He was successfully resuscitated and stabilized then transferred to the catheterization laboratory. Coronary angiogram revealed significant narrowing of the RCA at the previously diseased segment (Fig. 3), slightly improving after intra coronary nitroglycerin (Fig. 3). The LAD lesion was stable. Primary stenting of the RCA lesion was performed (Fig. 3). Furthermore, due to the potentially lethal ventricular arrhythmia that complicated the $R C A$ spasm, the relatively diffuse spasm on the RCA and the fear of future spasm in the non-stented segment, we decided to implant an intracardiac defibrillator (ICD). After an uneventful stay, the patient was discharged on calcium channel blockers (CCB) and dual antiplatelet therapy (DAPT).

Recently, and after uneventful seven years since the index episode, the patient presented to the emergency department with nine shocks delivered by the ICD, corresponding to a malignant ventricular arrhythmic storm and preceded by the same chest pain. Coronary angiogram was carried and showed diffuse RCA vasospasm partially subsiding with intra coronary nitroglycerin, which unmasked a significant narrowing upstream to the previous stent. Successful stenting of the target lesion was performed. Again, optimal medical treatment with maximally tolerated anti-vasospastic agents (CCB and nitro derivatives) and DAPT was pursued.

\section{Discussion}

CVS is infamous for its malignant, quite relatively uncommon, arrhythmogenic complications ${ }^{3,6,7}$. Recognizing and preventing this potential lethality with medical treatment and device therapy are a major cornerstone in the management of CVS patients ${ }^{8}$. The American Heart Association/American College of Cardiology/Heart Rhythm Society guidelines in 2017 have specifically addressed the issue of ICD placement in CVS patients ${ }^{9}$. ICD received a class IIa recommendation when medical therapy has failed in preventing CVS-induced SCD, and a class IIb recommendation in addition to medical therapy after a first episode of CVS-induced SCD. However, ICD remains under-utilized in this setting ${ }^{3}$.

Though uncommon, the presence of a "shark fin" pattern on ECG (Fig. 1), consisting of the blurring of the QRS and T-wave with significant ST-elevation, indicates a large burden of myocardial ischemia ${ }^{5}$. Known to be a high-risk pattern, this rare ECG finding predicts cardiogenic shock and mortality ${ }^{5}$. A recently published report showed that the "shark fin" pattern is associated with ventricular arrhythmias and cardiogenic shock in Takotsubo syndrome ${ }^{10}$. Miranda et al reported the case of a 53-year-old patient, where a transient "shark fin" pattern could have been caused by left main coronary spasm ${ }^{11}$.

Our case suggests that there may be an association between CVS-induced ventricular arrhythmias and certain ECG patterns such as the QRS-ST-T "shark fin" pattern. This specific pattern may predict cardiac sudden death during vasospasm. We hypothesize that this "shark fin" pattern, previously reported as a lifethreatening pattern in myocardial infarction ${ }^{4}$, may also indicate a lethal arrhythmogenic propensity in CVS patients. Therefore, if such a pattern is documented during CVS, we recommend implementing aggressive treatment strategies with medical and device therapy. Specifically, an ICD should be recommended despite stenting the potential target lesion, and that because of the diffuse and recurrent nature of CVS over time, as demonstrated in our observation where severe spasm recurred seven years after the initial episode.

\section{Conclusion}

CVS patients presenting with ventricular arrhythmias frequently continue to be treated medically with no device therapy. Our observation stresses on two issues. First, coronary spasm can be responsible for SCD. Second, a specific "shark fin" pattern on ECG may predict an arrhythmogenic propensity. Prompt recognition of this pattern while managing CVS patients and CVS-related ventricular arrhythmias should lower the ICD implantation threshold.

Conflict of interest: The Authors declare that there is no conflict of interest.

\section{References}


1. Prinzmetal M, Kennamer R, Merliss R, Wada T, Bor N. Angina pectoris. I. A variant form of angina pectoris; preliminary report. Am J Med . 1959; 27:375-388. doi:10.1016/0002-9343(59)90003-8

2. Lanza GA, Careri G, Crea F. Mechanisms of coronary artery spasm. Circulation . 2011;124(16):1774-1782. doi:10.1161/CIRCULATIONAHA.111.037283

3. Waldmann V, Bougouin W, Karam N, et al. Coronary Vasospasm-Related Sudden Cardiac Arrest in the Community. J Am Coll Cardiol . 2018;72(7):814-815. doi: 10.1016/j.jacc.2018.05.051

4. Asatryan B, Vaisnora L, Manavifar N. Electrocardiographic Diagnosis of Life-Threatening STEMI Equivalents: When Every Minute Counts. J Am Coll Cardiol Case Rep . 2019;1(4):666-668. doi: $10.1016 / j$.jaccas.2019.10.030

5. Cipriani A, D'Amico G, Brunello G, et al. The electrocardiographic "triangular QRS-ST-T waveform" pattern in patients with ST-segment elevation myocardial infarction: Incidence, pathophysiology and clinical implications. J Electrocardiol . 2018;51(1):8-14. doi: 10.1016/j.jelectrocard.2017.08.023

6. Myerburg RJ, Kessler KM, Mallon SM, et al. Life-threatening ventricular arrhythmias in patients with silent myocardial ischemia due to coronary-artery spasm. N Engl J Med . 1992;326(22):1451-1455. doi:10.1056/NEJM199205283262202

7. Sanna T, Lanza GA, Niccoli G, La Torre G, Cosentino N, Crea F. Coronary artery vasospasm causing ventricular fibrillation - an external loop recording. Resuscitation . 2009;80(4):393-394. doi: 10.1016/j.resuscitation.2008.12.013

8. Matsue Y, Suzuki M, Nishizaki M, Hojo R, Hashimoto Y, Sakurada H. Clinical implications of an implantable cardioverter-defibrillator in patients with vasospastic angina and lethal ventricular arrhythmia. $J$ Am Coll Cardiol . 2012;60(10):908-913. doi: 10.1016/j.jacc.2012.03.070

9. Al-Khatib SM, Stevenson WG, Ackerman MJ, et al. 2017 AHA/ACC/HRS guideline for management of patients with ventricular arrhythmias and the prevention of sudden cardiac death: Executive summary: A Report of the American College of Cardiology/American Heart Association Task Force on Clinical Practice Guidelines and the Heart Rhythm Society.Heart Rhythm . 2018;15(10): e190-e252. doi: 10.1016/j.hrthm.2017.10.035

10. Joki T, Nikus K, Laukkanen J. The electrocardiographic "triangular QRS-ST-T waveform" pattern: a marker of severe haemodynamic compromise in Takotsubo syndrome-a case report. Eur Heart J Case Rep . 2020;4(3):1-6. doi:10.1093/ehjcr/ytaa076

11. Miranda JM, de Oliveira WS, de Sá VP, de Sá IF, Neto NO. Transient triangular QRS-ST-T waveform with good outcome in a patient with left main coronary artery stenosis: A case report. $J$ Electrocardiol . 2019; 54:87-89. doi: 10.1016/j.jelectrocard.2019.02.002

\section{Abbreviations}

CCB: calcium channel blockers

CVS: coronary vasospasm

DAPT: dual antiplatelet therapy

ECG: electrocardiogram

FFR: fractional flow reserve

ICD: intracardiac defibrillator

LAD: left anterior descending

RCA: right coronary artery 
SCD: sudden cardiac death

TW: triangular QRS-ST-T waveform

VPCs: ventricular premature contractions

\section{Appendices}

Figure captions

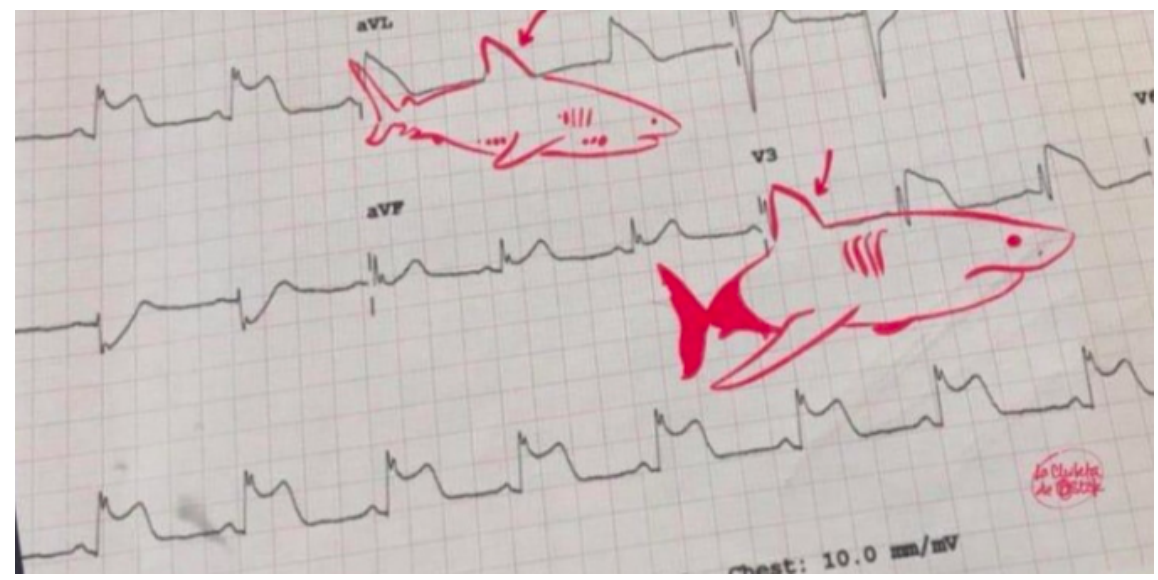

Figure 1. QRS-ST-T "shark fin" pattern.

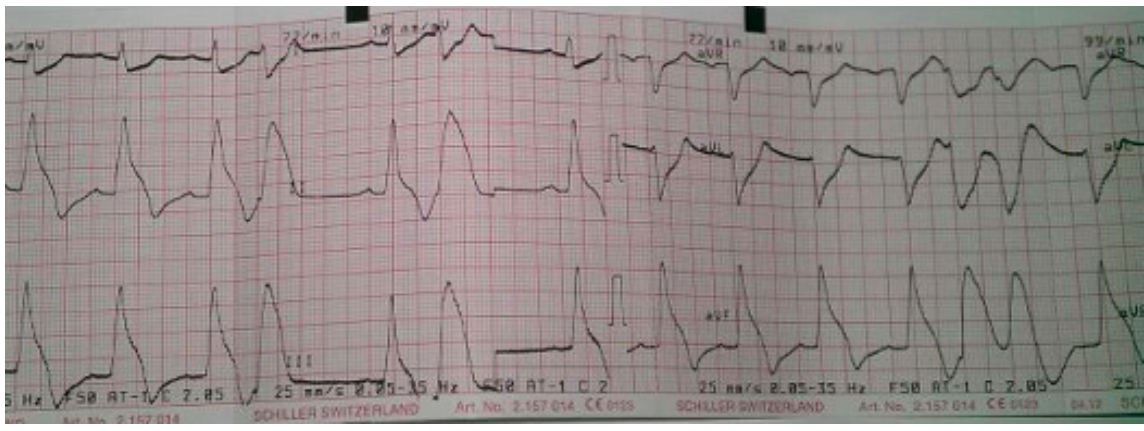

Figure 2. Inferior leads. QRS-ST-T "shark fin" pattern with frequent R/T VPCs

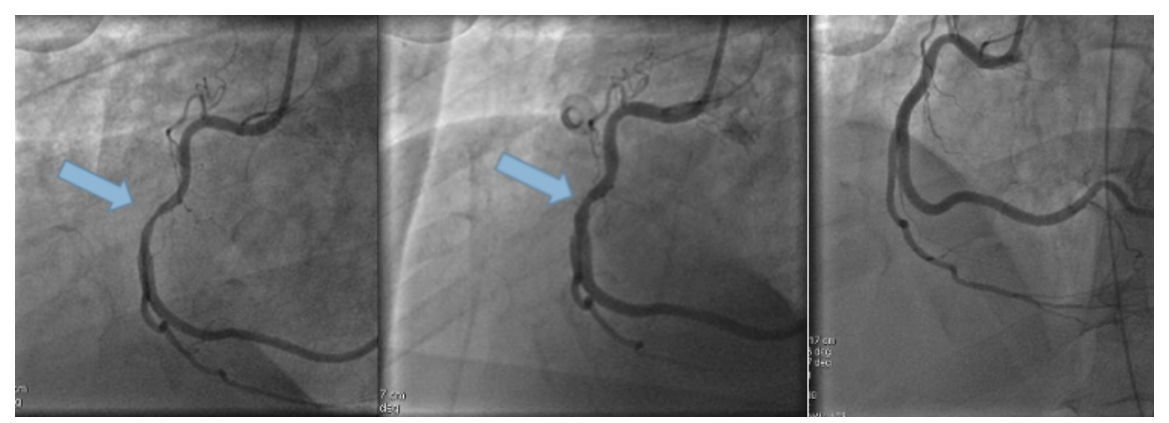

a b c 
Figure 3. Significant lesion in mid RCA (arrow in a) partially subsiding with intra coronary nitroglycerin (arrow in b). Final result after stenting of the target lesion shown in c. 